RESEARCH HIGHLIGHTS

AUTOIMMUNITY

\section{Spot the difference}

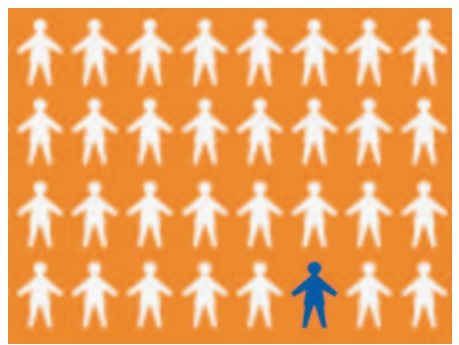

One possible explanation for the association between viral infections and autoimmune disease is molecular mimicry, in which foreign antigens activate $\mathrm{T}$ cells that crossreact with self-antigens. But how similar do the foreign and self-antigens have to be for the crossreaction to lead to clinical disease? Two papers by Ohashi and colleagues and von Herrath and colleagues show that the affinity of the self-reactive T-cell receptor (TCR) for the foreign antigen is an important factor in regulating the initiation of autoimmunity.

Gronski et al. used a mouse model of diabetes in which the lymphocytic choriomeningitis virus glycoprotein (LCMV-gp) is expressed as a 'selfantigen' under the control of the rat insulin promoter (RIP-gp) by pancreatic $\beta$-cells; these mice were crossed with P14 mice, which express a transgenic TCR specific for the gp33 epitope of LCMV-gp presented by $\mathrm{H}-2 \mathrm{D}^{\mathrm{b}}$. Immunization of $\mathrm{P} 14 \times \mathrm{RIP}-$ gp mice with wild-type LCMV results in $\beta$-cell destruction and diabetes. They looked at the ability of two LCMV variants (crossreacting 'foreign antigens') LCMV-L6F and LCMV-C4Y, which each have an amino-acid substitution in the most important LCMVgp epitope - to induce disease in this system.

Both variants bound $\mathrm{H}-2 \mathrm{D}^{\mathrm{b}}$; however, LCMV-L6F had 5-fold lower affinity for the P14 TCR than did wild-type LCMV-gp, and LCMV-C4Y had $\sim 20$-fold lower affinity. After immunization of P14 mice, wild-type LCMV and LCMV-L6F led to similar changes in activation-marker expression and cytotoxic activity by $\mathrm{P} 14$
T cells, although LCMV-L6F led to less T-cell proliferation. The lower-affinity LCMV-C4Y resulted in efficient but reduced cytotoxic activity. These differences in T-cell activation and proliferation properties were reflected in differences in disease induction in P14 $\times$ RIP-gp mice infected with the LCMV variants. All mice infected with wild-type LCMV develop diabetes, but only half of the mice infected with the medium-affinity variant, LCMV-L6F, developed disease. None of the mice immunized with the low-affinity variant, LCMV-C4Y, became diabetic.

Christen et al. provide similar evidence that, unless the crossreactive antigen is of sufficient affinity for the self-reactive TCR, disease is not initiated. They used a RIP-NP $\mathrm{H}-2^{\mathrm{b}}$ mouse model, in which LCMV nucleoprotein is expressed in the pancreas and diabetes is induced in 95\% of mice by infection with LCMV. However, infection with Pichinde virus (PV) - which has a crossreactive $\mathrm{NP}_{205}$ epitope that shares six out of eight amino acids with LCMV$\mathrm{NP}_{205}$ and binds $\mathrm{H}-2 \mathrm{~K}^{\mathrm{b}}$ with similar affinity - failed to induce diabetes. This is consistent with the $\sim 100$-fold lower avidity of PV-NP ${ }_{205}$ compared with LCMV-NP ${ }_{205}$ for the crossreactive TCR. But, although low-affinity mimics could not initiate disease in either study, this paper showed that they can accelerate an ongoing autoimmune process when PV infection occurs 1 month after LCMV infection in RIP-NP mice, by selectively expanding the LCMV-NP ${ }_{205}$-specific T-cell population in the pancreas.

Both studies therefore indicate that molecular mimicry might be unlikely to initiate autoimmunity, except in the case of rare high-affinity mimics. The second study shows that mimicry might have a more important role in accelerating disease in susceptible individuals with pre-existing inflammation of the target organ, and the first study also showed that defects in negative regulation, such as through CBL-B, could contribute to susceptibility.

\section{Kirsty Minton \\ (D) References and links ORIGINAL RESEARCH PAPERS Gronski, M. A} et al. TCR affinity and negative regulation limit autoimmunity. Nature Med. 10, 1234-1239 (2004) ] Christen, $U$ et al. A viral epitope that mimics a self antigen can accelerate but not initiate autoimmune diabetes. J. Clin. Invest. 114, 1290-1298 (2004).

\section{IN BRIEF}

\section{CELL MIGRATION}

Role of CCR8 and other chemokine pathways in the migration of monocyte-derived dendritic cells to lymph nodes.

Qu, C. et al. J. Exp. Med. 200, 1231-1241 (2004).

Monocytes can differentiate into either macrophages or lymphnode-homing dendritic cells (DCs); however, the events and molecules that are involved in monocyte fate are unclear. Although CCR2 is a key mediator of monocyte migration from the peritoneal cavity to the lymph nodes, in this study, the authors show that migration from the skin is CCR2 independent. In the skin, the GR $1^{\text {int }}$ mouse monocyte subpopulation readily differentiated into lymph-node-homing DCs and selectively expressed CCR7 and CCR8, which were both shown to be required for the emigration of these cells to the lymph nodes. CCR8 was also crucial for the migration of human monocytederived cells in a model of transendothelial trafficking, indicating a novel role for this chemokine in migration to lymph nodes.

\section{IMMUNODEFICIENCY}

\section{Severe combined immunodeficiency caused by} deficiency in either the $\delta$ or $\varepsilon$ subunit of CD3.

De Sainte Basile, G. et al. J. Clin. Invest. 114, 1512-1517 (2004).

This study identifies the genetic defects in patients with a form of severe combined immunodeficiency (SCID) that is characterized by a complete defect of peripheral T cells but normal B cells and natural killer cells. The molecular bases of other forms of SCID include mutations in the genes that encode cytokine receptors, components of factors involved in the generation of antigen receptors, the phosphatase CD45 and the $\delta$-chain of CD3. In this study, the authors show that mutations in the $C D 3 \delta$ or $C D 3 \varepsilon$ gene that lead to a deficiency in expression of these $\mathrm{CD} 3$ chains are the basis for the disease. Absence of $\mathrm{CD} 3 \delta$ was shown to cause a block in T-cell development at the intermediate stage of single-positive to double-positive transition.

\section{ANTIGEN PROCESSING}

\section{Unanticipated antigens: translation initiation at} CUG with leucine.

Schwab, S. R. et al. PLoS Biol. 2, 1774-1784 (2004).

Previous studies indicate that some MHC-class-I-bound peptides result from cryptic translation and that some of these peptides lack conventional AUG start codons. Schwab et al. investigated the mechanisms by which CUG functions as an initiation codon that encodes leucine. They found that the Kozak context of the CUG initiation codon influenced the efficiency with which it was decoded as leucine and that ribosomes specifically scan for the CUG initiation codon, indicating that CUG functions as a true initiation codon. However, the ribosomes that scan for CUG are probably distinct from those that scan for conventional AUG initiation codons, and these ribosomes use distinct factors to initiate translation, initiating translation with leucine independently of eukaryotic translation initiation factor $2 \alpha$. 